\title{
Étude des négociations du sens dans un tandem par visioconférence
}

\section{Marco Cappellini et Meng Zhang}

\section{OpenEdition}

Journals

Édition électronique

URL : http://journals.openedition.org/rdlc/1576

DOI : $10.4000 /$ rdlc. 1576

ISSN : 1958-5772

Éditeur

ACEDLE

Référence électronique

Marco Cappellini et Meng Zhang, «Étude des négociations du sens dans un tandem par visioconférence ", Recherches en didactique des langues et des cultures [En ligne], 10-2 | 2013, mis en ligne le 07 juin 2013, consulté le 01 mai 2019. URL : http://journals.openedition.org/rdlc/1576 ; DOI : $10.4000 /$ rdlc. 1576

Ce document a été généré automatiquement le 1 mai 2019.

\section{cc) $(1) \odot$}

Recherches en didactique des langues et des cultures is licensed under a Creative Commons AttributionNonCommercial-NoDerivatives 4.0 International License 


\title{
Étude des négociations du sens dans un tandem par visioconférence
}

\author{
Marco Cappellini et Meng Zhang
}

\section{Introduction}

1 Des connexions Internet à haut débit et des logiciels de visioconférence ${ }^{1}$ toujours plus performants ont ouvert de nouvelles possibilités pour des échanges authentiques entre apprenants habitant différents coins de la planète. Cet article considère l'une de ces possibilités, le télétandem (Telles, 2009) et ses potentialités pour l'acquisition des langues étrangères. Nous adoptons la perspective du cadre input-interaction ${ }^{2}$ en l'élargissant en fonction des études sur la multimodalité dans la Communication Médiatisée par Ordinateur (CMO). Notre but principal est de comprendre quelles sont les caractéristiques de l'acquisition des langues en télétandem telles qu'elles se révèlent dans les routines de négociation du sens (NdS).

2 Dans cette introduction, nous aborderons dans un premier temps la question de l'apprentissage des langues en télétandem. Ensuite, nous allons nous pencher sur le cadre input-interaction et les études sur la CMO qui se situent dans ce cadre. L'introduction se termine avec l'illustration de certaines études sur la visioconférence dans l'apprentissage des langues autour de la notion d'affordance communicative. Suite à l'introduction, nous aborderons notre cadre méthodologique et illustrerons les données analysées. La troisième section sera consacrée aux résultats de l'étude, discutés dans la quatrième section.

\section{L'apprentissage des langues en (télé)tandem}

3 L'apprentissage des langues en tandem est une méthode pédagogique où deux apprenants de langue maternelle différente et étudiant chacun la langue de l'autre interagissent pour s'entraider dans leurs apprentissages (Helmling, 2002). Les partenaires interagissent la 
moitié du temps dans une langue et l'autre moitié dans l'autre, en prenant en alternance les rôles d'apprenant et d'usager expert de la langue parlée. À ce propos, Telles (2009) formule l'hypothèse d'une "symétrie dynamique" dans les échanges. Cette symétrie associe les avantages des interactions entre apprenants et de celles avec un locuteur expert: d'une part, cela permet l'instauration d'un environnement non menaçant pour les "faces" des apprenants (Varonis \& Gass, 1985); d'autre part, il y a une très faible possibilité d'"incorrections" (Gass \& Varonis, 1994).

4 L'apprentissage des langues en tandem est basé sur les principes de l'autonomie de l'apprenant et de la réciprocité (Helmling, 2002). Le principe d'autonomie implique que les apprenants sont responsables de leur apprentissage et peuvent donc établir leurs objectifs d'apprentissage et choisir comment les atteindre. Le principe de réciprocité implique que l'aide reçue quand on prend le rôle d'apprenant est liée à l'aide offerte quand on prend le rôle d'usager expert.

5 Les premiers tandems documentés remontent aux années 1960 et se déroulaient en présentiel (Helmling, 2002). Ensuite, pendant les années 1990, le tandem a été appliqué à différents dispositifs de CMO écrite et a pris le nom de e-Tandem (Brammerts, 1996). Le télétandem est l'application du tandem dans des dispositifs de CMO par visioconférence ${ }^{3}$ (Telles, 2009).

\section{Le cadre input-interaction}

6 Dans le domaine des Recherches en Acquisition des Langues (RAL), l'approche appelée cadre input-interaction (Gass, 1997) se base sur l'hypothèse interactionniste (Interaction hypothesis) formulée par Long (1983a, 1983b) et enrichie par les études de Varonis et Gass (1985) et Swain $(1985,1995)$, jusqu'à sa formulation dans Long (1996).

7 L'hypothèse interactionniste reprend et élargit l'hypothèse de l'input (Input Hypothesis. Krashen, 1985) en affirmant que pour qu'il y ait acquisition chez l'apprenant, celui-ci doit non seulement être exposé à un input compréhensible, notamment l'input modifié du xénolecte (Long, 1983a), mais aussi il doit pouvoir interagir afin de rendre l'input compréhensible par des ajustements interactifs, appelés interaction modifiée (Long, 1983b). Ces ajustements interactifs ont été étudiés notamment en termes de négociations du sens (NdS) (Varonis \& Gass, 1985).

Les NdS apparaissent quand il y a une suspension de la progression de l'interaction à cause d'une incompréhension. La NdS est alors une séquence latérale par laquelle les interlocuteurs résolvent le problème de compréhension avant de reprendre la discussion. Les NdS sont révélatrices des premières phases de l'acquisition dans la mesure où elles permettent à l'apprenant le repérage (noticing) de décalages entre son interlangue et la langue cible, ce qui est une condition nécessaire, bien que non suffisante, de l'acquisition (Schmidt, 1990).

9 Dans le cas de l'interaction modifiée, le problème à résoudre est l'incompréhension par l'apprenant d'un tour de parole de l'interlocuteur. Dans le contexte du télétandem, nous parlerons dans ce cas de NdS déclenchée par un tour de parole de l'usager expert.

En ce qui concerne la structure d'une NdS, nous gardons le modèle de Varonis et Gass (1985). Nous sommes conscients de l'existence d'une adaptation de ce modèle à la CMO par Smith (2003). Néanmoins, cette adaptation est strictement liée à la nature écrite de la CMO écrite synchrone, raison pour laquelle nous gardons le modèle de Varonis et Gass. 
Nous sommes également conscients que ce modèle a été initialement développé pour l'étude d'interactions entre locuteurs non-natifs (terminologie de Varonis et Gass), mais des recherches successives ont prouvé son efficacité dans l'étude d'autres interactions (voir infra).

11 Le modèle de Varonis et Gass (1985) identifie quatre éléments principaux : le déclencheur (trigger), l'indicateur (indicator) - ensuite appelé signal (signal) (Pica, 1994) - la réponse (response) et la réaction à la réponse (reaction to response), qui est optionnelle. Le déclencheur est un élément langagier énoncé par $\mathrm{A}$ qui pose problème à $\mathrm{B}$. Le signal est un élément langagier, paralinguistique et/ou posturo-mimo-gestuel (Cosnier et Develotte in Develotte et al. 2011) par lequel $\mathrm{B}$ signale à $\mathrm{A}$ qu'il y a un problème. La réponse est ce que $\mathrm{A}$ énonce ou fait afin de résoudre le problème. Enfin, la réaction à la réponse est ce que B éventuellement dit pour signaler que le problème est résolu. Chacun des éléments de la résolution peut à son tour devenir un déclencheur générant une $\mathrm{NdS}$ complexe (expanded).

Dans le cadre input-interaction, l'hypothèse interactionniste est complétée par l'hypothèse de l'output (Output Hypothesis) de Swain (1985), selon laquelle pour qu'il y ait acquisition chez l'apprenant, celui-ci doit non seulement pouvoir négocier l'input, mais aussi produire en L2 pour pouvoir restructurer son interlangue si nécessaire. Autrement dit, si la production est jugée incompréhensible ou non recevable par l'interlocuteur, celui-ci pourra donner une rétroaction négative à l'apprenant suite à laquelle l'apprenant sera amené à formuler une production poussée (pushed output) ou "modifiée". La rétroaction négative peut être soit explicite soit implicite (Long, 1996). La première consiste à pointer explicitement une erreur, alors que la rétroaction implicite signale l'erreur sans la pointer ouvertement, par exemple par une reformulation.

Comme l'interaction modifiée, les épisodes amenant à une production modifiée peuvent être analysés à l'aide du modèle de Varonis et Gass. Nous distinguons deux types de NdS en fonction du déclencheur de la négociation. D'un côté, Swain (1995) note que, pendant son tour de parole, l'apprenant peut être confronté à un manque, par exemple un mot inconnu en langue cible, qui déclenche une NdS visant à trouver le mot en question. Nous parlerons dans ce cas de NdS déclenchée par un manque dans un tour de parole de l'apprenant. De l'autre côté, une expression incorrecte de l'apprenant pourrait déclencher chez l'usager expert une rétroaction négative. Dans ce cas, nous parlerons de NdS déclenchée par un tour de parole de l'apprenant non conforme à la langue cible.

\section{Etudes des rétroactions négatives et négociations du sens dans la CMO}

14 Le cadre input-interaction a été mobilisé de différentes façons par des chercheurs s'intéressant à la CMO (Chapelle, 2009 et Kern, 2006). La plupart des études se sont intéressées à la CMO écrite synchrone par clavardage en binômes d'apprenants (Blake, 2000, Lee, 2008, Pellettieri, 2000, Sheraky \& Tahririan, 2006, Smith, 2003) ou entre un apprenant et un locuteur natif ${ }^{4}$ (Tudini, 2003). D'autres types de CMO étudiés dans cette perspective sont les MOOs (Kotter, 2003, O’Rourke, 2005), l'audioconférence (Jepson, 2005) et la visioconférence (Nicolaev, 2010, Wang, 2006).

Les résultats montrent que la $\mathrm{CMO}$ écrite facilite chez les apprenants le repérage (noticing) (Blake, 2000, Smith, 2003). De plus, le texte demeurant accessible tout au long de 
l'interaction, les études ont mis en relief l'absence complète de répétitions ${ }^{5}$ dans les routines de NdS (Kotter, 2003, Sarré, 2011), contrairement à l'audioconférence (Jepson, 2005) ou la visioconférence (Sarré, 2011).

En ce qui concerne les rétroactions, plusieurs études montrent que les rétroactions explicites sont moins courantes que celle implicites (Bower et Kawagichi 2011, Kotter 2003, Sarré 2011 entre autres). Des raisons possibles sont le caractère menaçant pour la face des apprenants des rétroactions négatives explicites (Lee in Belz \& Thorne, 2006, Sarré, 2011), ou, dans des e-Tandems, la réticence des interlocuteurs à prendre un rôle d'enseignant (Kotter, 2003, Bower \& Kawaguchi, 2011). Enfin, les études montrent que les déclencheurs sont très majoritairement lexicaux.

\section{CMO par visioconférence et affordances communicatives}

Dans toute CMO, d'une part, la technologie devient l'un des acteurs de l'interaction (Liddicoat in Develotte et al. 2011) ; d'autre part, elle entre en interaction avec les autres dimensions, notamment celle sociale (Lamy, 2008).

Pour prendre en considération la dimension technologique des interactions en télétandem, en suivant Develotte et al. (2011) et Lamy \& Hampel (2007), nous reprenons les concepts d'affordance (Gibson, 1979) et d'affordance communicative (Hutchby, 2001). Une affordance est ce qu'un environnement offre à un agent impliqué dans une action. Une affordance communicative sera ce qui permet aux interlocuteurs d'instaurer un certain type d'interaction dans un dispositif de communication, indépendamment des usages prévus par les concepteurs. Les logiciels de visioconférence présentent plusieurs affordances communicatives manipulables par les interlocuteurs : la modalité vidéo, la modalité audio et la modalité écrite ${ }^{6}$. La co-construction de sens par les interlocuteurs se fait dans une « orchestration » de ces différentes modalités (Hauck, 2010).

Quant à la relation entre les affordances communicatives de la visioconférence et les routines de négociation, Nicolaev (2010) et Wang (2006) font l'hypothèse que les différentes modalités, en fonctionnant en synergie, ont un impact sur les routines. Plus spécifiquement, Nicolaev (2010) montre que la multimodalité joue un rôle important dans les négociations du sens au niveau des indicateurs - parfois réalisés à la seule aide de la mimique - et dans les réponses - par exemple par l'utilisation exclusive des gestes ou du clavardage.

Cette brève revue de la littérature nous permet d'affiner notre problématique et de poser nos questions de recherche:

21 1. Quelles sont les caractéristiques des routines des trois types de NdS ?

22 2. Est-ce que le principe de réciprocité donne effectivement lieu à une symétrie dynamique (Telles, 2009) dans les NdS? Autrement dit, est-ce que les routines de NdS varient d'une langue à l'autre?

23 3. Comment les participants exploitent-ils les affordances de la visioconférence dans les routines de NdS en télétandem? 


\section{Contexte et méthode}

\section{Contexte et données}

24

$$
\begin{aligned}
& \text { inspiré du Teletandem Brazil } \text {, le Télétandem Dalian-Lille. Il s'agit d'un projet de } \\
& \text { télécollaboration concernant des étudiants chinois en troisième année de licence }{ }^{8} \text { de } \\
& \text { français à l'Université de Langues Etrangères de Dalian (Chine), et des étudiants en } \\
& \text { première et deuxième année de master à l'UFR de Langues Étrangères Appliquées de } \\
& \text { l'Université Lille } 3 \text { SHS (France). Tous les étudiants ont un niveau de maîtrise de la langue } \\
& \text { étrangère étudiée correspondant au B1, avec des rares B2, sur l'échelle du Cadre Européen } \\
& \text { Commun de Référence (Conseil de l'Europe, 2001). }
\end{aligned}
$$

Le parcours des étudiants se divise en trois phases: une première phase où, conformément au principe d'autonomie, chaque étudiant est amené à se fixer des objectifs et un plan d'apprentissage par un entretien conseil (Gremmo, 1995). La deuxième phase consiste en cinq sessions de télétandem de deux heures chacune. La troisième phase, qui clôt le parcours, consiste en un entretien d'autoévaluation avec un tuteur.

Les données sont constituées d'enregistrements audio et vidéo collectés par les étudiants chinois avec l'accord de leurs partenaires francophones. La transcription a été principalement orthographique avec des annotations portant sur la multimodalité.

Cet article est une étude de cas sur un corpus de quatre sessions télétandem entre deux apprenantes - L et S - interagissant par Skype ${ }^{9}$. L est une étudiante chinoise de 21 ans ayant étudié le français en Chine pendant deux ans et ayant atteint le niveau B1+. S est une étudiante française de 23 ans qui a étudié le chinois pendant trois ans, dont un en Chine, et ayant atteint le niveau B1. Les deux apprenantes ont réalisé leurs sessions télétandem de leurs domiciles.

L'ensemble des interactions analysées est de sept heures et trente minutes ${ }^{10}$ pour un total de 5147 tours de parole (tdp) et 59200 mots environ.

\section{Méthode}

Notre objectif est d'analyser les dynamiques de l'acquisition dans les routines de NdS. Notre approche analytique est foncièrement quantitative.

Dans un premier temps, nous avons identifié et classé les trois types de NdS qui nous intéressent: les NdS déclenchées par un tdp de l'usager expert (Long, 1983b), les NdS déclenchées par un tdp de l'apprenant présentant un manque (Swain, 1995), les NdS déclenchées par un tdp de l'apprenant non conforme à la langue cible (Swain, 1985).

Ensuite, nous avons classé les tdp de chaque type de NdS en distinguant les composantes du modèle de Varonis et Gass : déclencheur, signaux, réponses et réactions aux réponses. Pour chaque composante, nous avons classé et comptabilisé le type de tdp en exploitant la typologie de Kotter (2003), adaptée et enrichie en fonction de notre contexte en nous basant sur les études de Lyster et Ranta (1997) et Nicolaev (2010). Pour rendre compte des affordances communicatives de la visioconférence, nous avons ajouté notamment des catégories correspondant à l'utilisation indépendante d'un mode différent de celui oral.

Recherches en didactique des langues et des cultures, 10-2 | 2013 
Les types de tdp sont reproduits dans l'annexe 2 avec une description et un exemple pour chacun.

\section{Résultats}

Dans cette section, premièrement nous donnons une vue d'ensemble des interactions analysées; deuxièmement, nous analysons les trois types de $\mathrm{NdS}$; troisièmement, nous nous focaliserons sur l'exploitation des affordances communicatives.

\section{Les négociations du sens dans les interactions télétandem}

Le tableau 1 fournit deux types d'information : les premières deux lignes donnent une vue d'ensemble de la distribution de la parole dans les sessions; les lignes suivantes portent sur les NdS. La parole est distribuée de manière relativement équilibrée, avec une légère prépondérance de l'étudiante chinoise. En effet, elle parle $54 \%$ de la session en chinois et $51 \%$ en français, contre $46 \%$ en chinois et $49 \%$ en français pour l'étudiante française.

TABLEAU 1 - Vue d'ensemble des NdS dans les interactions télétandem

\begin{tabular}{|c|c|c|c|c|c|c|c|c|c|c|}
\hline & chn 1 & $\operatorname{chn} 2$ & $\operatorname{chn} 3$ & $\operatorname{chn} 4$ & Moyenne chn & fil & fr2 & $\mathrm{ft} 3$ & fr 4 & Moyenne fr \\
\hline $\begin{array}{l}\text { Tdp de lusager expert } \\
\text { (nombre pourcentage } \\
\text { de la session) }\end{array}$ & $\begin{array}{l}407 / \\
53,9 \%\end{array}$ & $\begin{array}{l}360 \% \\
52,2 \%\end{array}$ & $\begin{array}{l}173 / \\
53,6 \%\end{array}$ & $\begin{array}{l}466 \\
157,2 \%\end{array}$ & $\begin{array}{ll}\mathbf{5 4 , 2 2 5 \%} & \text { du } \\
\text { total } & \text { des } \\
\text { sessions } & \text { en } \\
\text { chinois } & \end{array}$ & $\begin{array}{l}303 / \\
50,8 \%\end{array}$ & $\begin{array}{l}330 / \\
46,4 \%\end{array}$ & $\begin{array}{l}375 i \\
50,3 \%\end{array}$ & $\begin{array}{l}240 \\
47 \%\end{array}$ & $\begin{array}{l}48,625 \% \text { du } \\
\text { total des } \\
\text { sessions en } \\
\text { français }\end{array}$ \\
\hline $\begin{array}{l}\text { Tdp de lapprenant } \\
\text { (nombre pourcentage } \\
\text { de la session) }\end{array}$ & $\begin{array}{l}348 / \\
46,1 \%\end{array}$ & $\begin{array}{l}330 \% \\
47,8 \%\end{array}$ & $\begin{array}{l}150 / \\
46,4 \%\end{array}$ & $\begin{array}{l}349 / \\
42,8 \%\end{array}$ & $\begin{array}{ll}45,775 \% & \text { du } \\
\text { total } & \text { des } \\
\text { sessions } & \text { en } \\
\text { chinois } & \end{array}$ & $\begin{array}{l}294 \\
49,2 \%\end{array}$ & $\begin{array}{l}381 / \\
53,6 \%\end{array}$ & $\begin{array}{l}370 / \\
49,7 \%\end{array}$ & $\begin{array}{l}271 / \\
53 \%\end{array}$ & $\begin{array}{l}51,375 \% \text { du } \\
\text { total des } \\
\text { sessions en } \\
\text { français }\end{array}$ \\
\hline $\begin{array}{l}\text { Nombre total des NdS } \\
\text { (nombre total des } \\
\text { tdppourcentage de la } \\
\text { session) }\end{array}$ & $\begin{array}{l}34 \\
(395 / \\
52,3 \%)\end{array}$ & $\begin{array}{l}25 \\
(295 / \\
42,7 \%)\end{array}$ & $\begin{array}{l}20 \\
(141 \\
/ 43,7 \%)\end{array}$ & $\begin{array}{l}21 \\
(307 / \\
37,7 \%)\end{array}$ & $\begin{array}{l}25 \text { NdS par } \\
\text { session } \\
\text { (les NdS } \\
\text { courrent } \\
44,1 \% \text { des } \\
\text { tours de } \\
\text { l'interaction) }\end{array}$ & $\begin{array}{l}21 \\
(224 / \\
37,5 \%)\end{array}$ & $\begin{array}{l}15 \\
(232 / \\
32,6 \%)\end{array}$ & $\begin{array}{l}14 \\
(127 / \\
17 \%)\end{array}$ & $\begin{array}{l}30 \\
(213 / \\
41,7 \%)\end{array}$ & $\begin{array}{l}20 \text { NoM par } \\
\text { session } \\
\text { (les NdS } \\
\text { courrent } \\
32,2 \% \text { des } \\
\text { tours de } \\
\text { linteraction) }\end{array}$ \\
\hline $\begin{array}{l}\text { NdS déclenchées par } \\
\text { un tdp non-conforme } \\
\text { de lapprenant }\end{array}$ & 9 & 7 & 2 & 4 & (22 totales) & 11 & 5 & 7 & 11 & (34 totales) \\
\hline $\begin{array}{l}\text { NdS déclenchées par } \\
\text { un todp avec un manque } \\
\text { de l'apprenant }\end{array}$ & 14 & 5 & 3 & 5 & (27 totales) & 4 & 9 & 3 & 16 & (32 totales) \\
\hline $\begin{array}{l}\text { NdS déclenchés par un } \\
\text { tdp de lusager expert }\end{array}$ & 11 & 13 & 15 & 12 & (51 totales) & 6 & 1 & 4 & 3 & (14 totales) \\
\hline
\end{tabular}

Le nombre moyen de NdS est plus important dans les échanges en chinois : $25 \mathrm{NdS}$ ( $44 \%$ de la session) par session en chinois contre $20 \mathrm{NdS}$ (32\%) par session en français. En chinois, le plus grand nombre de NdS est déclenché par des tdp de l'usagère experte (51), alors que dans les parties en français il s'agit de NdS déclenchées par un tdp de l'apprenante non conforme à la langue cible (34) ou présentant un manque (32). 


\section{Les routines des négociations du sens}

Le tableau 2 présente les éléments les plus fréquents dans ce type de $\mathrm{NdS}^{12}$. La colonne de
gauche indique la phase de la NdS et la fréquence ( $1=$ le plus fréquent ; $2=$ le deuxième
plus fréquent). Les deux autres colonnes explicitent quels sont les types de tdp les plus
fréquents et quel est le pourcentage de présence dans le cadre de la même phase de la
NdS. En cas de parité de pourcentages, plusieurs types sont présentés.

Le tableau 2 présente les éléments les plus fréquents dans ce type de $\mathrm{NdS}^{12}$. La colonne de
gauche indique la phase de la NdS et la fréquence ( $1=$ le plus fréquent ; $2=$ le deuxième
plus fréquent). Les deux autres colonnes explicitent quels sont les types de tdp les plus
fréquents et quel est le pourcentage de présence dans le cadre de la même phase de la
NdS. En cas de parité de pourcentages, plusieurs types sont présentés.

Le tableau 2 présente les éléments les plus fréquents dans ce type de $\mathrm{NdS}^{12}$. La colonne de
gauche indique la phase de la NdS et la fréquence ( $1=$ le plus fréquent ; $2=$ le deuxième
plus fréquent). Les deux autres colonnes explicitent quels sont les types de tdp les plus
fréquents et quel est le pourcentage de présence dans le cadre de la même phase de la
NdS. En cas de parité de pourcentages, plusieurs types sont présentés.

Le tableau 2 présente les éléments les plus fréquents dans ce type de $\mathrm{NdS}^{12}$. La colonne de
gauche indique la phase de la NdS et la fréquence ( $1=$ le plus fréquent ; $2=$ le deuxième
plus fréquent). Les deux autres colonnes explicitent quels sont les types de tdp les plus
fréquents et quel est le pourcentage de présence dans le cadre de la même phase de la
NdS. En cas de parité de pourcentages, plusieurs types sont présentés.

TABLEAU 2 - NdS déclenchées par un tdp de l'usagère experte

\begin{tabular}{|l|l|l|}
\hline élément & français & chinois \\
\hline Déclencheur 1 & Lexique (71,4\%) & Lexique (80,4\%) \\
\hline Déclencheur 2 & Prononciation (21,4\%) & Prononciation et ton (19,6\%) \\
\hline Signal 1 & Demande de clarification (56,2\%) & Demande de clarification (39,8\%) \\
\hline Signal 2 & Hétéro-répétition (18,8\%) & Hétéro-répétition (31,4\%) \\
\hline Réponse 1 & Paraphrase (44,8\%) & Paraphrase (42,5\%) \\
\hline Réponse 2 & $\begin{array}{l}\text { Auto-répétition / } \\
\text { réponse minimale / } \\
\text { vérification de compréhension (13,8\%) }\end{array}$ & Alternance codique (23,3\%) \\
\hline
\end{tabular}

$\mathrm{S}:[\ldots]$ en France il y a un cliché

L : cliché ?

( $\mathrm{s}:$ un cliché)

(S: un stéréotype)

$\mathrm{S}:$ tu comprends?

$\mathrm{L}$ : euh oui oui devient donc déclencheur de la NdS. L'apprenante produit une hétéro-répétition au tdp suivant. L'usagère experte paraphrase le mot en fournissant un synonyme et renforce sa paraphrase par deux envois par clavardage. Enfin, elle produit une vérification de compréhension. Ces quatre tdp constituent la réponse. L'apprenant clôt la $\mathrm{NdS}$ avec une réponse minimale affirmative.

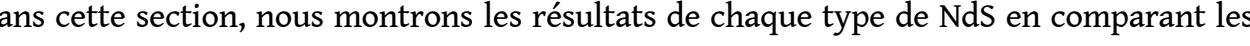
deux langues. Pour chaque type de NdS, nous présentons et analysons un exemple et Les NdS déclenchées par un tdp de l'usagère experte

Les NdS déclenchées par un tdp de l'usagère experte correspondent à ce que Long (1983b) appelle l'interaction modifiée. En voici un exemple ${ }^{11}$ : 


\begin{tabular}{|l|l|l|}
\hline Réaction à la réponse 1 & Accusé de réception (50\%) & Accusé de réception (59,7\%) \\
\hline Réaction à la réponse 2 & Alternance codique / prise (20\%) & Prise (29,8\%) \\
\hline
\end{tabular}

encheurs sont principalement lexicaux pour les deux langues. Quant aux signaux, dans les deux cas le plus utilisé est la demande de clarification, suivie de la répétition. La réponse la plus fréquente est la paraphrase. Notons que si cela est une stratégie efficace en français, pendant les échanges en chinois la paraphrase génère d'autres problèmes de communication et par conséquent des $\mathrm{NdS}$ complexes. Une autre différence est l'utilisation de l'alternance codique (code switching) en chinois, ce qui indique que face à l'inefficacité de la paraphrase, l'usagère experte décide de s'exprimer en français. Enfin, les réactions aux réponses sont très présentes même si elles sont optionnelles. L'accusé de réception est la plus fréquente d'entre elles, suivi de la prise ${ }^{13}$ par l'apprenant $\mathrm{du}$ déclencheur de la NdS.

\section{Les NdS déclenchées par un manque dans le tdp de l'apprenante}

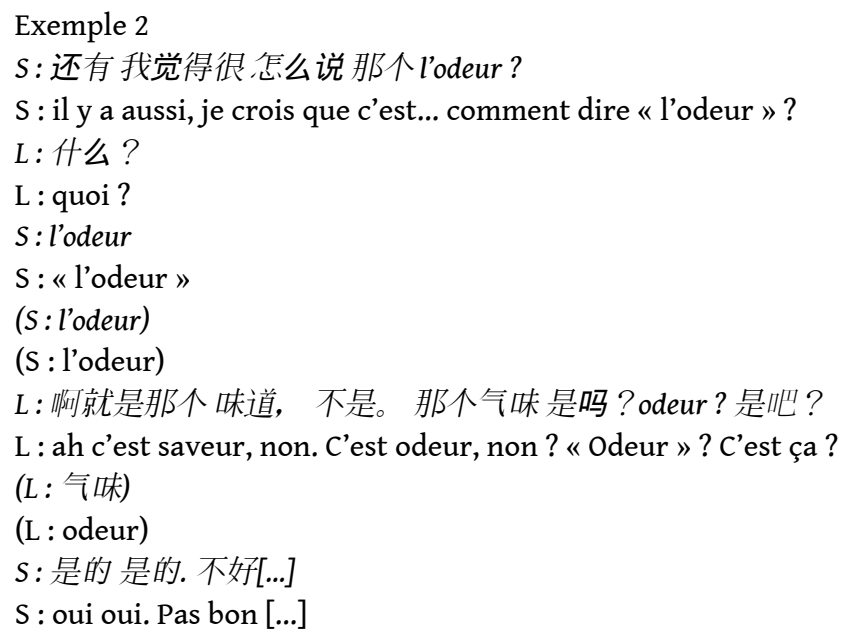

Face à un manque pendant son tdp (Swain, 1995), l'apprenante déclenche une NdS en changeant de langue pour se faire fournir un item lexical (odeur) en L2. Le déclencheur est donc lexical et le signal est une alternance codique, où l'apprenante emploie le mot français "odeur» pour signaler son manque de lexique et demander la traduction chinoise. L'usagère experte ne comprend pas immédiatement la question et produit une demande de clarification au niveau de la réponse. L'apprenante répète alors le mot dans sa L1 à l'oral et à l'écrit. Ensuite, l'usagère experte produit trois réponses: une paraphrase, une demande de confirmation et un envoi par clavardage. Enfin, l'apprenante produit une réponse minimale et reprend la conversation.

TABLEAU 3 - NdS déclenchées par un manque dans le tdp de l'apprenante

\begin{tabular}{|l|l|l|}
\hline élément & français & chinois \\
\hline Déclencheur 1 & Lexique (96,7\%) & Lexique (100\%) \\
\hline
\end{tabular}




\begin{tabular}{|l|l|l|}
\hline Déclencheur 2 & Prononciation (3,3\%) & / \\
\hline Signal 1 & Demande de confirmation (39,6\%) & Alternance codique (44\%) \\
\hline Signal 2 & Demande d'aide langagière (32,1\%) & Demande d'aide langagière (28\%) \\
\hline Réponse 1 & Paraphrase (32\%) & Paraphrase (38,9\%) \\
\hline Réponse 2 & Réponse minimale (18\%) & Alternance codique (26,9\%) \\
\hline Réaction à la réponse 1 & Accusé de réception (54,5\%) & Prise (53,8\%) \\
\hline Réaction à la réponse 2 & Prise (45,5\%) & Accusé de réception (38,5\%) \\
\hline
\end{tabular}

Les déclencheurs sont presque exclusivement lexicaux. Les routines sont différentes dans les deux langues. En chinois, le signal le plus employé est l'alternance codique: l'apprenante dit un mot en français afin de demander sa traduction en chinois (exemple 2). D'autre part, en français, le signal le plus utilisé est la demande de confirmation: l'apprenante énonce un mot dont elle n'est pas sûre avec une intonation montante et attend ensuite que sa partenaire confirme (ou non) le mot employé. Pour les réponses, la paraphrase est la plus fréquente dans les deux langues. Ensuite, en français on trouve la réponse minimale, produite notamment suite aux demandes de confirmations au niveau du signal. En chinois, c'est l'alternance codique qui est largement présente. Toujours en chinois, la réaction à la réponse la plus fréquente est la prise de la part de l'apprenante. D'autre part, en français on trouve l'accusé de réception (acknowledgement).

\section{Les NdS déclenchées par un tdp de l'apprenante non conforme à la langue cible}

Le troisième type de NdS analysé est celui déclenché par un tour de l'apprenante non conforme à la langue cible. Comme les négociations précédentes, ces $\mathrm{NdS}$ amènent l'apprenant à une production poussée (Swain, 1985). En voici un exemple :

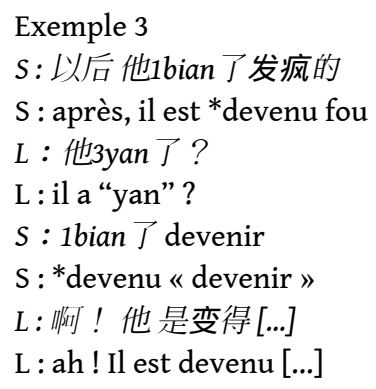

43 Au début de la négociation, l'apprenante produit un mot erroné dans la mesure où elle utilise un premier ton ${ }^{14}$ au lieu du quatrième. Le déclencheur est classé « ton ». Cela cause une incompréhension de la part de l'usagère experte, qui reformule en produisant une demande de confirmation au niveau du signal. La réponse de l'apprenante est une répétition suivie d'une alternance codique. L'usagère experte reformule à nouveau. Notons par ailleurs que cette négociation se poursuit outre la question du ton de变 pour corriger l'expression 变发疯, également incorrecte ${ }^{15}$. 
TABLEAU 4 - NdS déclenchées par un tdp de l'apprenante non-conforme

\begin{tabular}{|l|l|l|}
\hline élément & français & chinois \\
\hline Déclencheur 1 & Lexique (47,2\%) & Lexique (46,7\%) \\
\hline Déclencheur 2 & Morphosyntaxe (41,7\%) & Ton (36,7\%) \\
\hline Signal 1 & Reformulation (47,5\%) & Explication (32\%) \\
\hline Signal 2 & $\begin{array}{l}\text { Demande de clarification / hétéro-répétition } \\
(16,4 \%)\end{array}$ & Reformulation (21,3\%) \\
\hline Réponse 1 & Production modifiée (34,4\%) & $\begin{array}{l}\text { Production } \\
(37,6 \%)\end{array}$ \\
\hline Réponse 2 & Réponse minimale (27,9\%) & Auto-répétition (17,6\%) \\
\hline Réaction à la réponse & Accusé de réception (80,8\%) & $\begin{array}{l}\text { Accusé de réception } \\
(89,7 \%)\end{array}$ \\
\hline 1 & Réaction à la réponse \\
2 & Rassurement (11,5\%) & Non-verbal (6,9\%) \\
\hline
\end{tabular}

Les déclencheurs sont toujours majoritairement lexicaux, mais cette fois avec une présence importante d'autres types. En chinois, le second déclencheur le plus fréquent est le ton; en français il s'agit de la morphosyntaxe. Les routines diffèrent dans les deux langues concernant les signaux. En chinois, le plus fréquent est l'explication, suivie par les reformulations $\left(\right.$ recast $\left.^{16}\right)$. En français, le signal le plus employé est la reformulation, suivie de la répétition et la demande de clarification. La réponse la plus fréquente est la production modifiée dans les deux langues. Enfin, la réaction à la réponse la plus fréquente est l'accusé de réception.

\section{La multimodalité dans les négociations}

Une analyse qualitative et quantitative de la multimodalité amène à plusieurs observations. Avant tout, les éléments non-verbaux et paraverbaux ne peuvent être considérés comme des tours de parole à part entière que rarement (19 fois). Les deux cas les plus fréquents sont: 1 . l'usagère experte fait oui de la tête comme "accusé »; 2 . l'apprenante sourit pour signaler qu'elle ne comprend pas, comme dans le début de $\mathrm{NdS}$ suivant :

\section{Exemple 4}




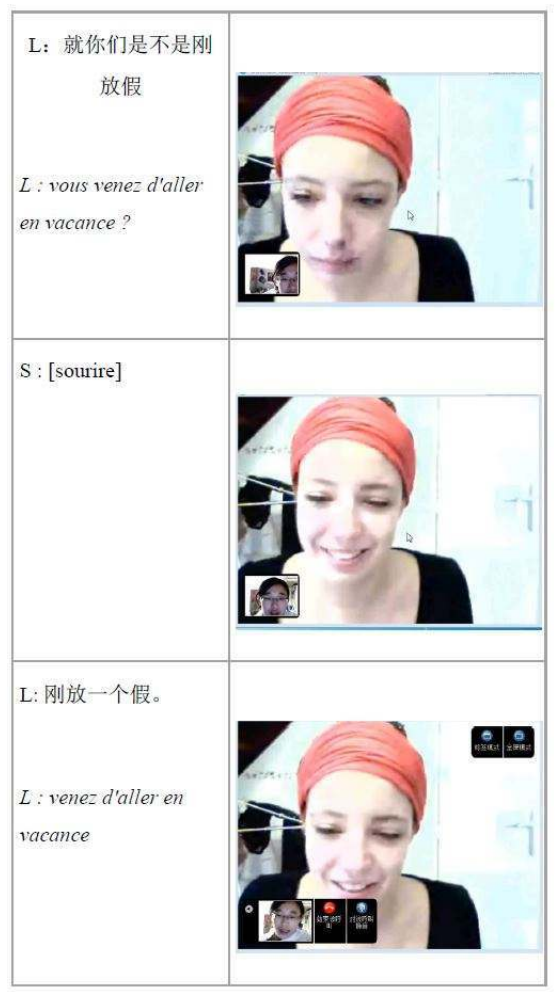

La modalité vidéo devient une affordance pour communiquer à travers des mimiques et des gestes. De plus, elle devient en deux occurrences une affordance pour montrer du chinois écrit en pinyin sur un papier quand les apprenantes ne savaient pas comment intégrer les accents symbolisant les tons dans le clavardage.

En ce qui concerne la modalité écrite, les messages forment un nombre de tdp quantitativement plus important que les non- et para-verbaux. En chinois, cette modalité constitue une affordance pour écrire en caractères aussi bien qu'en pinyin, selon les besoins du moment. Le tableau 6 montre le recours au clavardage en relation à : 1 . dans quel type de NdS il a lieu ; 2. à quel niveau de la NdS le clavardage est employé ; 3 . à quel type de tdp il est associé.

Tableau 5 - Le clavardage dans les NdS

\begin{tabular}{|c|c|c|c|c|c|}
\hline $\begin{array}{l}\text { NdS déclenchées par un tdp de l'usager } \\
\text { expert dans la session chinoise }\end{array}$ & $\mathbf{T}$ & S & $\begin{array}{l}\mathbf{R} \\
\text { auto-répétition } 1 \\
\text { paraphrase } 20 \\
\text { alternance } \\
\text { codique } 9\end{array}$ & RR & $\begin{array}{l}\text { Total } \\
30\end{array}$ \\
\hline $\begin{array}{l}\text { NdS déclenchées par un manque } \\
\text { langagier dans le tdp de l'apprenant } \\
\text { dans la session chinoise }\end{array}$ & $\mathbf{T}$ & $\begin{array}{l}\mathrm{S} \\
\text { alternance codique } \\
9 \\
\text { paraphrase } 1 \\
\text { demande d'aide } \\
\text { langagière } 14\end{array}$ & $\begin{array}{l}\mathbf{R} \\
\text { demande de } \\
\text { clarification } \\
1 \\
\text { hétéro-répétition } \\
3 \\
\text { paraphrase } 7\end{array}$ & $\mathbf{R R}$ & $\begin{array}{l}\text { Total } \\
35\end{array}$ \\
\hline
\end{tabular}




\begin{tabular}{|c|c|c|c|c|c|}
\hline $\begin{array}{l}\text { NdS déclenchées par un tdp non- } \\
\text { conforme de l'apprenant dans la session } \\
\text { chinoise }\end{array}$ & $\mathbf{T}$ & $\begin{array}{l}\text { S } \\
\text { Demande de } \\
\text { confirmation } 3 \\
\text { reformulation } 6 \\
\text { explication18 }\end{array}$ & $\mathbf{R}$ & $\mathbf{R R}$ & $\begin{array}{l}\text { Total } \\
27\end{array}$ \\
\hline $\begin{array}{l}\text { NdS déclenchées par un tdp de l'usager } \\
\text { expert dans la session française }\end{array}$ & $T$ & S & $\begin{array}{l}\mathbf{R} \\
\text { auto-répétition } 1 \\
\text { paraphrase } 2\end{array}$ & $\mathbf{R R}$ & $\begin{array}{l}\text { Total } \\
3\end{array}$ \\
\hline $\begin{array}{l}\text { NdS déclenchées par un manque } \\
\text { langagier dans le tdp de l'apprenant } \\
\text { dans la session française }\end{array}$ & $\mathbf{T}$ & $\begin{array}{l}\text { S } \\
\text { demande d'aide } \\
\text { langagière } 3 \\
\text { alternance codique } \\
5 \\
\text { demande de } \\
\text { confirmation } 2\end{array}$ & $\begin{array}{l}\mathbf{R} \\
\text { hétéro-répétition } \\
1 \\
\text { paraphrase } 7 \\
\text { alternance } \\
\text { codique } 2 \\
\text { clavardage } 3 \\
\text { reformulation } 2\end{array}$ & $\mathbf{R R}$ & $\begin{array}{l}\text { Total } \\
25\end{array}$ \\
\hline $\begin{array}{l}\text { NdS déclenchées par un tdp non- } \\
\text { conforme de l'apprenant dans la session } \\
\text { française }\end{array}$ & $\mathbf{T}$ & $\begin{array}{l}\text { S } \\
\text { reformulation } 2\end{array}$ & \begin{tabular}{|l}
$\mathbf{R}$ \\
auto-répétition 2 \\
paraphrase 2 \\
production \\
modifiée 1
\end{tabular} & $\mathbf{R R}$ & $\begin{array}{l}\text { Total } \\
7\end{array}$ \\
\hline
\end{tabular}

$\mathrm{Ni}$ les déclencheurs ni les réactions à la réponse ne sont constitués ou suivis par le clavardage. Le clavardage est utilisé surtout dans les parties en chinois, en marge d'une paraphrase orale au niveau de la réponse dans une $\mathrm{NdS}$ déclenchée par un tdp de l'usagère experte ( 20 fois), ou en relation à une explication au niveau du signal dans une $\mathrm{NdS}$ déclenchée par un tdp de l'apprenante non conforme à la langue cible (18 fois). Une fois que le terme ou l'expression est inscrit dans l'espace de clavardage, il demeure à disposition pendant l'interaction. Nos données confirment l'hypothèse que cette disponibilité est exploitée pour des prises, éventuellement multiples, dans la suite de l'interaction.

Enfin, nous avons noté une utilisation problématique de la modalité orale lors des échanges en chinois. Quand l'apprenante française n'est pas sûre d'un mot, elle emploie parfois une intonation montante qui constituerait une demande de confirmation. Si cela est une stratégie efficace en français, en chinois l'adoption d'une telle intonation change le ton du mot et peut causer des NdS.

\section{Discussion}

Nos analyses montrent que les interactions télétandem permettent la présence de NdS, principalement lexicales. En comparant nos résultats avec d'autres études, on note que le pourcentage des NdS est sensiblement plus haut dans nos données. En effet, la moyenne totale de NdS est d'environ $38 \%$ des interactions, alors qu'il s'agissait de 30,4\% dans l'étude de Kotter (2003) sur des tandems interagissant par MOO ; 7,2\% en anglais et $20 \%$ en japonais dans l'étude de Bower et Kawaguchi (2011) sur des tandems apprenant par 
clavardage ${ }^{17}$; environ $15 \%$ dans Nicoalev (2010) et 2,9\% dans Sarré (2011) pour la CMO par visioconférence. Cela semble suggérer que la quantité des NdS est liée plus au type d'interaction qu'au dispositif technique. Autrement dit, l'absence d'une tâche communicative débouchant sur une production semble permettre une quantité majeure de NdS, ce qui confirme les observations de Kasper (2004) sur les conversations-pourapprendre. Cette donnée nous semble importante d'un point de vue didactique, surtout si l'objectif d'un dispositif est de permettre aux apprenants de négocier le sens par l'interaction.

51 D'autre part, nos résultats vont à l'encontre, en partie, de ceux de O'Rourke (2005) et de Bower et Kawaguchi (2011) à propos de l'impact du niveau de compétence en langue sur les NdS. D'après ces auteurs, un niveau inférieur causerait une plus grande quantité de rétroactions. Or, notre étude suggère que le niveau n'a pas un lien causal avec la quantité de rétroactions, car même si les niveaux des apprenantes sont similaires, les quantités de NdS diffèrent : 44,1 \% en chinois contre 32,2\% en français. L'explication de ces décalages pourrait être à rechercher non seulement dans des éventuelles différences de niveau, mais aussi dans les rôles discursifs pris par les interlocuteurs.

52 Notre étude rejoint les autres (cf. 1.3) à propos des déclencheurs des $\mathrm{NdS}$, de nature principalement lexicale. Par contre, la distinction entre différents types de NdS nous a permis de constater que cette prédominance est en partie contrebalancée dans le cas des NdS déclenchées par un tour de parole de l'apprenante non-conforme à la langue cible, avec $41,7 \%$ de déclencheurs morphosyntaxiques en français et $36,7 \%$ de déclencheurs constitués par le ton en chinois. Notre étude confirme également que même si la réaction à la réponse est un élément optionnel, elle est très largement présente dans les interactions par visioconférence (Nicolaev, 2010, Wang, 2006).

53 En ce qui concerne la réalisation du principe de réciprocité dans les routines de $\mathrm{NdS}$, les analyses nous amènent à mettre en question l'hypothèse d'une "symétrie dynamique" (Telles, 2009). Avant tout, le type de NdS le plus fréquent est différent dans les deux langues : en chinois il y a un nombre nettement supérieur de NdS déclenchées par un tour de parole de l'usagère experte, alors qu'en français, il y a un nombre similaire de $\mathrm{NdS}$ déclenchées par un tour de l'apprenant présentant un manque ou un énoncé non conforme à la langue cible (tableau 1). Ensuite, les routines ne sont pas les mêmes (tableaux 2, 3 et 4). Dans les NdS déclenchées par un tdp de l'usagère experte, les routines diffèrent au niveau des réponses et, par conséquent, des réactions aux réponses: en chinois, il y a un recours fréquent à l'alternance codique suite aux paraphrases, souvent inefficaces. L'alternance codique distingue également les $\mathrm{NdS}$ déclenchées par un manque dans le tdp de l'apprenante. Dans ces NdS, en français l'apprenante chinoise essaie de prononcer un mot et demande confirmation alors que celle française demande directement de l'aide, souvent avec une alternance codique. Autrement dit, l'interprétation du rôle d'apprenante est différente pour les deux étudiantes : l'étudiante chinoise prend plus de risques dans la conversation que l'étudiante française. Enfin, les NdS déclenchées par un tdp de l'apprenante non conforme à la L2 sont différentes au niveau du signal : en position d'usagère experte, l'étudiante française utilise largement la reformulation, alors que l'étudiante chinoise produit davantage d'explications.

54 Ces observations nous amènent à préciser que la réciprocité due à l'alternance des rôles d'apprenante et d'usagère experte ne débouche pas sur une symétrie dynamique dans la réalisation discursive de ces rôles. Nous pensons qu'une étude de la réalisation discursive 
des rôles en télétandem est nécessaire pour mieux comprendre la réalisation du principe de réciprocité.

Notre étude nous a permis également de prendre en compte la multimodalité des interactions télétandem. Plusieurs chercheurs ont discuté de l'utilité de la modalité vidéo en visioconférence, sans trouver un consensus. Ainsi Wang (2004) affirme que par rapport à des dispositifs d'audio-conférence, il n'existe aucun gain observable. Au contraire, Develotte et al. (2010) ou Yamada et Akahori (2009) soutiennent que la vidéo rend l'intercompréhension plus facile. Sans entrer dans un débat comparatif, dans notre corpus la modalité vidéo devient une affordance dans le cadre des $\mathrm{NdS}$, permettant l'intégration d'éléments mimiques et posturo-gestuels dans l'interaction, ce qui confirme les résultats de Nicolaev (2010).

Les analyses confirment que la modalité écrite est hautement intégrée dans les conversations (Develotte et al., 2008) et qu'elle a différentes fonctions, les principales étant de clarifier ce qui a été dit ou de corriger des erreurs (ibid., Guichon \& Drissi, 2008). De plus, l'écrit restant disponible tout au long des conversations, il permet des prises retardées. Enfin, dans les NdS, l'écrit n'est jamais employé comme déclencheur ou réaction à la réponse.

L'articulation entre les trois types de NdS permet de détailler différentes observations sur l'impact des NdS sur l'acquisition du chinois et du français (annexe 3). Dans les NdS déclenchées par un tdp de l'usagère experte, dans les échanges en français, 10 déclencheurs lexicaux amènent à 4 prises, alors qu'en chinois 41 déclencheurs lexicaux donnent lieu à 20 prises. Dans les NdS déclenchées par un manque dans le tdp de l'apprenante, 27 déclencheurs en chinois amènent à 21 prises, alors qu'en français 32 déclencheurs amènent à 15 prises. De manière générale donc, face à un nouveau élément langagier, surtout lexical, dans nos données l'apprenante opère une prise la moitié des fois. Enfin, pour ce qui est des NdS déclenchées par un tdp de l'apprenante non conforme à la langue cible (annexe 3), en français 36 déclencheurs amènent à 21 productions modifiées, tandis qu'en chinois 30 déclencheurs amènent à 32 productions modifiées ${ }^{18}$.

Même si ces observations ne permettent pas de tirer des conclusions concernant l'acquisition à long terme, elles suggèrent que les interactions télétandem peuvent promouvoir l'acquisition dans ses premières phases. En effet, soit par une interaction modifiée (Long 1983b), soit par une production poussée (Swain, 1985), les apprenants opèrent des repérages souvent suivis de prises ou de productions modifiées.

\section{Conclusions}

Notre étude nous amène à une série de considérations. Avant tout, les interactions télétandem favorisent la présence de NdS, notamment lexicales, dont la quantité est beaucoup plus grande que les autres études recensées. La quantité de NdS ne semble pas être liée à l'utilisation de la visioconférence, mais au contraire au type d'interaction : le (télé)tandem. En effet, il semble que comme les apprenants n'ont pas une production à réaliser et à rendre, ils prennent le temps pour mener les négociations de sens, parfois longues, afin d'atteindre l'intercompréhension. Par conséquent, il nous semble possible suggérer que si la négociation du sens rentre dans leurs objectifs pédagogiques, au moment de l'élaboration des tâches les formateurs pourraient trouver pertinent de prévoir des moments de conversation-pour-apprendre (Kasper, 2004). 
60 réalisation du principe de réciprocité dans les $\mathrm{NdS}$. Les résultats montrent que les routines diffèrent dans les deux langues, autrement dit que les NdS ne sont pas symétriques. Nous avons fait l'hypothèse que cela est lié à des différences dans l'interprétation des rôles d'apprenante et d'usagère experte. Des recherches à venir portant sur des corpus plus importants pourront mieux étudier cette hypothèse.

61 fonction des besoins de la communication, en en faisant des affordances communicatives. Le sens est négocié majoritairement à travers une orchestration des modalités (Hauck, 2010), par exemple l'écrit comme soutien à l'oral. Les analyses ont également montré que la modalité vidéo permet l'intégration d'éléments posturo-mimo-gestuels (Cosnier et Develotte in Develotte et al. 2011). Par conséquent, il nous semble qu'un formateur voulant permettre à ses étudiants de communiquer (aussi) à travers ces éléments dans un dispositif de télécollaboration aurait intérêt à considérer l'utilisation de la visioconférence, bien que cela implique plus de questions techniques que d'autres dispositifs de CMO.

Pour conclure, cette étude présente une vue d'ensemble des NdS dans les sessions télétandem d'un binôme. Nos résultats demandent à être confrontés à des études sur des corpus plus larges, études en cours de réalisation. De plus, une étude plus approfondie sur le corpus du présent article pourrait montrer l'évolution de l'utilisation des modalités et plus largement des routines de négociation au fil des sessions, ce qui permettrait de formuler des hypothèses quant au développement des compétences interactionnelles et multimodales des apprenants.

\section{BIBLIOGRAPHIE}

Belz, J. \& Thorne, S. L. (eds.) (2006). Internet-mediated Intercultural Foreign Language Education, Boston: Thomson and Heinle.

Blake, R. (2000). "Computer mediated communication: a window to L2 Spanish interlanguage". Language Learning and Technology, vol. 4, $\mathrm{n}^{\circ}$ 1, pp. 120-136.

Bower, J. \& Kawaguchi, S. (2011). "Negotiation of meaning and corrective feedback in Japanese/ English eTandem". Language Learning and Technology, vol. 15, n 1, pp. 41-71.

Brammerts, H. (1996). "Language learning in tandem using the internet". In M. Warschauer (ed.), Telecollaboration in foreign language learning. Honolulu: Second Language Teachng and Curriculum Center.

Chapelle, C. A. (2009). "The relationship between second language acquisition theory and computer-assisted language learning". Modern Language Journal, vol. 93, pp. 741-753.

Conseil de l'Europe (2001). Cadre européen commun de référence pour les langues. Paris : Didier. 
Develotte, C., Guichon, N. \& Kern R. (2008). "'Allo Berkeley ? Ici Lyon... Vous nous voyez bien ?" Étude d'un dispositif de formation en ligne synchrone franco-américain à travers le discours de ses usagers". Alsic, vol. 11, pp. 129-156.

Develotte, C., Guichon, N. \& Vincent, C. (2010). "The use of the webcam for teaching a foreign language in a desktop videoconferencing environment", ReCALL, vol. 22, n 3, pp. 293-212

Develotte, C., Kern R. \& Lamy, M.-N. (eds.) (2011). Décrire la conversation en ligne. Le face à face distanciel. Lyon: ENS Editions.

Firth, A. \& Wagner, J. (1997). "On Discourse, Communication, and (Some) Fundamental Concepts in SLA Research", Modern Language Journal, vol. 81, n³, pp. 285-300.

Gass, S. M. (1997). Input, Interaction, and the Second Language Learner. New York, London: Routledge. Gass, S. M. \& Varonis, E. M. (1994). "Input, Interaction, and Second Language Production". Studies in Second Language Acqusition, vol. 16, pp. 283-302.

Gibson, J. J. (1979). The Ecological Approach to Visual Perception. London: Lawrence Erlbaum Associates.

Gremmo, M.-J. (1995). "Conseiller n'est pas enseigner". Mélanges CRAPEL, vol. 22, pp. 33-61.

Guichon, N. \& Drissi, S. (2008). "Tutorat de langue par visioconférence : comment former aux régulations pédagogiques ?". Les Cahiers de l'Acedle, vol. 5, pp. 185-217.

Hauck, M. (2010). "Telecollaboration: At the interface between multimodal and intercultural communicative competence". In S. Guth \& F. Helm (ed.). Telecollaboration 2.0. Oxford: Peter Lang. Helmling, B. (dir.) (2002). L'apprentissage autonome des langues en tandem. Paris: Didier.

Hutchby, I. (2001). Conversation and technology: from the telephone to the Internet. Cambridge: Polity. Jepson, K. (2005). "Conversation - and negotiated interaction - in text and voice chat rooms". Language Learning and Technology, vol. 9, $\mathrm{n}^{\circ}$ 3, pp. 79-98.

Kasper, G. "Participant orientation in German conversation-for-learning". Modern Language Journal, vol. 88, n 4, pp. 551-567.

Kern, R. (2006). "La communication médiatisée par ordinateur en langues. Recherches et applications récentes aux USA". In C. Dejean-Thircuir \& F. Mangenot (dir.) Les échanges en ligne dans l'apprentissage et la formation. Paris: Clé.

Kotter, M. (2003). "Negotiation of meaning and codeswitching in online tandems". Language Learning and Technology, vol. 7, $\mathrm{n}^{\circ} 2$, pp. 145-172.

Krashen, S. (1985). The input hypothesis: issues and implications. Harlow : Longman.

Lamy, M.-N. (2008). "Ce que cyber-parler veut dire : quels cadres théoriques pour l'analyse des conversations multimodales en réseau ?". In J. Gerbault (dir.). La Langue du Cyberespace : de la diversité aux normes. Paris: L'Harmattan.

Lamy, M.-N. \& Hampel R. (2007). Online communication in language learning and teaching. New York: Palgrave MacMillan.

Lee, L. (2008). "Focus-on-form through collaborative scaffolding in expert-to-novice online interaction". Language Learning and Technology, vol. 12, n 3, pp. 53-72.

Long, M. H. (1983a). "Linguistic and conversational adjustments to non-native speakers". Studies in Second Language Acquisition, vol. 5, pp. 177-193. 
Long, M. H. (1983b). "Native speaker/Nonnative speaker Conversation and the Negotiation of Comprehensible input". Applied Linguistics, vol. 4, pp. 126-141.

Long, M. H. (1996). "The role of the linguistic environment in second language acquisition". In W. Ritchie \& T. K. Bhatia (eds). Handbook of second language acquisition. San Diego, CA: Academic Press.

Lyster, R. \& Ranta, L. (1997) "Corrective feedback and learner uptake. Negotiation of form in communicative classrooms". Studies in Second Language Acquisition, vol. 20, pp. 37-66.

Macaire, D. (2004). "Du Tandem au Télé-Tandem. Nouveaux apprentissages, nouveaux outils, nouveaux rôles". Consulté en septembre 2012 http://www.tele-tandem.net/fr/wp-content/ uploads/2010/02/Macaire-Du-tandem-au-Tele-Tandem.pdf

Mangenot, F. (2006). "Glossaire". In C. Dejean-Thircuir, F. Mangenot (dir.), Les échanges en ligne dans l'apprentissage et la formation. Paris : Clé.

Nicolaev, V. (2010). "Les négociations de sens dans un dispositif d'apprentissage des langues synchrone par visioconférence". Les Cahiers de l'Acedle, vol. 7, n 2, pp. 169-198.

O’Rourke, B. (2005). "Form-focused interaction in online tandem learning". Calico journal, vol. 22, pp. 433-466.

Pellettieri, J. (2000). "Negotiation in cyberspace: the role of chatting in the development of grammatical competence". In M. Warschauer \& R. Kern (eds.) Network-based language teaching: concepts and practice. Cambridge: Cambridge University Press.

Pica, T. (1994). "Research on Negotiation: What does it reveal about second language learning conditions, processes, and outcomes?" . Language Learning, vol. 44, pp. 493-527.

Sarré, C. (2011). "Computer-Mediated Negotiated Interactions: How is Meaning Negotiated in Discussion Boards, Text Chat and Videoconferencing?". In S. Thouesny \& L. Bradley (eds.), Second Language Teaching and Learning with Technology. Dublin: Research-publishing.net.

Schmidt, R. (1990). "The role of consciousness in second language learning". Applied Linguistics, vol. 11, pp. 129-158.

Sheraky, M. \& Tahririan, M. H. (2006). "Negotiation of Meaning and Noticing in text-Based Online Chat". Modern Language Journal, vol. 90, pp. 557-573.

Smith, B. (2003). "Computer-Mediated Negotiated Interaction: An Expanded Model". Modern Language Journal, vol. 87, $\mathrm{n}^{\circ} 1$, pp. 38-57.

Sotillo, S. (2000). "Discourse Functions and Syntactic Complexity in Synchronous and Asynchronous Communication". Language Learning and Technology, vol. 4, n 1, pp. 82-119.

Swain, M. (1985). "Communicative competence: Some roles of comprehensible input and comprehensible output in its development". In S. Gass \& C. Madden (eds.), Input in Second Language Acquisition. Rowley: Newbury House.

Swain, M. (1995). "Three functions of output in second language learning". In G. Cook \& B. Seidlhofer (eds.), Principle and Practice in Applied Linguistics. Oxford : Oxford University Press. Telles, J. A. (ed.) (2009). Teletandem. Um contexto virtual, autônomo, colaborativo para aprendizagem das linguas estrangeiras no século XXI. Campinas, SP: Pontes Editores.

Tudini, V. (2003). "Using native speakers in chat". Language Learning and Technology, vol. 7, n 3, pp. 141-159.

Varonis, E. M. \& Gass, S. (1985). "Non-native/ non-native conversation. A model for negotiation of meaning". Applied Linguistics, vol. 6, pp. 71-90. 
Véronique, G. D. (2011). "Le traitement de l'input dans l'acquisition des langues étrangères". Langage, Interaction et Acquisition (LIA), vol. 1, pp. 159-164.

Wang, Y. (2004). "Supporting synchronous distance language learning with desktop videoconferencing". Language Learning and Technology, vol. 8, n 3, pp. 90-121.

Wang, Y. (2006). "Negotiation of meaning in desktop videoconferencing-supported distance language learning". ReCALL, vol. 18, pp. 122-164.

Yamada, M. \& Akahori, K. (2009). "Awareness and performance through self- and partner's image in videoconferencing". Calico Journal, vol. 27, pp. 1-25.

\section{ANNEXES}

\section{Convention de transcription}

\begin{tabular}{|l|l|}
\hline$:$ & allongement \\
\hline (texte) & $\begin{array}{l}\text { message de clavardage. La position dans la transcription est celle de l'apparition du } \\
\text { message dans le logiciel de visioconférence }\end{array}$ \\
\hline$*$ & élément langagier incorrect \\
\hline 1bian & transcription pinyin d'un mot incompréhensible. Le numéro indique le ton prononcé \\
\hline
\end{tabular}

\section{Typologie des tours de parole dans les négociations du sens}

Dans les exemples, les parties en gras mettent en évidence le tour décrit, les parties en italique sont des traductions du chinois. « $\mathrm{D}$ » indique « déclencheur »; « $\mathrm{S}$ » correspond à « signal »; « $\mathrm{R}$ » renvoie à « réponse »; « $R \mathrm{R}$ » signifie « réaction à la réponse ».

\begin{tabular}{|l|l|l|}
\hline Tour & Description & Exemple \\
\hline $\begin{array}{l}\text { Accusé de réception } \\
\text { Acknowledgement) }\end{array}$ & $\begin{array}{l}\text { C'est un tour de RR. Le locuteur ayant } \\
\text { produit le signal affirme que la négociation } \\
\text { a été terminée avec succès et qu'il a } \\
\text { compris. }\end{array}$ & S : ah d'accord \\
\hline Alternance codique & $\begin{array}{l}\text { Lelle de la session (normalement la langue } \\
\text { maternelle du partenaire, parfois l'anglais) }\end{array}$ & $\begin{array}{l}\text { plus long c'est la Saint Jean? } \\
\text { euh } \\
\text { L : euh c'est c'est pas } \\
\text { c'est pas le sol- solstice } \\
\text { d'été ? }\end{array}$ \\
\hline
\end{tabular}




\begin{tabular}{|c|c|c|}
\hline $\begin{array}{l}\text { Assertion } \\
\text { d'incompréhension }\end{array}$ & $\begin{array}{l}\text { Le locuteur dit ouvertement qu'il n'a pas } \\
\text { compris ce que l'interlocuteur a dit. }\end{array}$ & $\begin{array}{l}\mathrm{L}: \text { 我没有听明白再说一 } \\
\text { 遍好吗? } \\
\text { L: je n'ai pas compris, tu } \\
\text { peux répéter? }\end{array}$ \\
\hline Auto-répétition & $\begin{array}{l}\text { Le locuteur répète ce qu'il a déjà dit sans } \\
\text { rien changer. }\end{array}$ & $\begin{array}{l}\mathrm{L}: \text { il était un poète et } \\
\text { aussi un policien } \\
\mathrm{S}: \text { et aussi un? } \\
\mathrm{L}: \text { policien policien } \\
\mathrm{S}: \text { policien ? un politicien } \\
\mathrm{L}: \text { ah oui oui oui } \\
\text { politicien [rire] }\end{array}$ \\
\hline $\begin{array}{l}\text { Demande } \\
\text { langagière }\end{array}$ & $\begin{array}{l}\text { Le locuteur affirme ne pas savoir comment } \\
\text { s'exprimer et éventuellement demande } \\
\text { explicitement comment le faire, mais sans } \\
\text { changer de langue (cf. Alternance } \\
\text { codique). }\end{array}$ & $\begin{array}{l}\text { L : c'est pas ça comment } \\
\text { dire . euh il il a coupé } \\
\text { une une ligne, il euh } \\
\text { attend elle elle a fait une } \\
\text { ligne pour se débarrasser }\end{array}$ \\
\hline $\begin{array}{l}\text { Demande } \\
\text { clarification }\end{array}$ & $\begin{array}{l}\text { Le locuteur demande à l'interlocuteur } \\
\text { qu'est-ce qu'il a dit. }\end{array}$ & $\begin{array}{l}\text { S : 什么? } \\
S: q u o i ?\end{array}$ \\
\hline \multirow[t]{2}{*}{$\begin{array}{l}\text { Demande } \\
\text { confirmation }\end{array}$} & $\begin{array}{l}\text { Dans les NdS déclenchées par un tour de } \\
\text { parole de l'usager expert, le locuteur } \\
\text { reformule ce que l'interlocuteur a dit et } \\
\text { demande si c'est ce que l'interlocuteur } \\
\text { voulait dire. }\end{array}$ & $\begin{array}{l}\text { S : c'est oui oui . et il y a } \\
\text { beaucoup de monde qui } \\
\text { fait de la chirurgie } \\
\text { esthétique en chine? } \\
\text { L: euh il y a beaucoup } \\
\text { de stars. c'est ça? }\end{array}$ \\
\hline & $\begin{array}{l}\text { Dans les NdS déclenchées par un manque } \\
\text { langagier, l'apprenant essaie le mot qu'il } \\
\text { pense être correct et demande } \\
\text { confirmation. }\end{array}$ & $\begin{array}{l}\text { L : et les gens il y a un, } \\
\text { dialecte c'est ça? }\end{array}$ \\
\hline Excuse & $\begin{array}{l}\text { Le locuteur s'excuse, normalement pour } \\
\text { une « erreur ». }\end{array}$ & L : déso- désolée \\
\hline Explication & $\begin{array}{l}\text { C'est un tour de } S \text { dans les NdS déclenchée } \\
\text { par un tour non-conforme de l'apprenant, } \\
\text { éventuellement après un feedback } \\
\text { correctif direct. L'usager expert explique } \\
\text { l'erreur et éventuellement suggère une } \\
\text { forme plus correcte. }\end{array}$ & $\begin{array}{l}\mathrm{L}: \text { 我们不说 不说古老 } \\
\text { 我们就说他们是很老的 } \\
\text { 歌手 } \\
\text { L: nous ne disons pas [un } \\
\text { chanteur] 'antique', nous } \\
\text { disons qu'il est un vieux } \\
\text { chanteur }\end{array}$ \\
\hline
\end{tabular}




\begin{tabular}{|c|c|c|}
\hline Hétéro-répétition & $\begin{array}{l}\text { Le locuteur répète, entièrement ou en } \\
\text { partie, l'intervention de l'interlocuteur, } \\
\text { souvent dans une question. }\end{array}$ & $\begin{array}{l}\text { L: il était un poète et } \\
\text { aussi un policien } \\
\mathrm{S}: \text { et aussi un? } \\
\mathrm{L}: \text { policien policien } \\
\mathrm{S}: \text { policien? un politicien } \\
\mathrm{L}: \text { ah oui oui oui } \\
\text { politicien [rire] }\end{array}$ \\
\hline $\begin{array}{l}\text { Non-verbal } \\
\text { (paralinguistique, } \\
\text { mimique et/ou } \\
\text { gestuel) }\end{array}$ & $\begin{array}{l}\text { Le locuteur utilise seulement une } \\
\text { expression paralinguistique, une mimique } \\
\text { et/ou un geste, souvent comme un S } \\
\text { d'incompréhension. }\end{array}$ & $\begin{array}{l}\mathrm{L}: \text { 这是谁给你找的 } \\
\text { 阠叮? } \\
\text { L:ça, qui te l'a trouvé? } \\
\mathrm{S}: \mathbf{m h} ?\end{array}$ \\
\hline Paraphrase & $\begin{array}{l}\text { C'est un tour de } \mathrm{R} \text { dans les } \mathrm{NdS} \\
\text { déclenchées par un tour de parole de } \\
\text { l'usager expert. L'usager expert élabore ce } \\
\text { qu'il a dit avec des mots différents. }\end{array}$ & $\begin{array}{l}\text { S: 卡是什么? 是carte? } \\
\text { S: }{ }^{19}{ }^{19} \text { 'est quoi? C'est } \\
\text { 'carte'? } \\
\text { L : euh 不是 卡就是说 } \\
\text { euh比如说euh怎么说呢 } \\
\text { ? euh 就是钥匙 钥匙呢 } \\
(\text {...) } \\
\text { L: non. 卡signifie, euh, par } \\
\text { exemple, euh, comment } \\
\text { dire... euh [si tu as] une } \\
\text { clé une clé qui (...) }\end{array}$ \\
\hline Prise & $\begin{array}{l}\text { L'apprenant répète l'élément qui lui } \\
\text { manquait et l'intègre éventuellement dans } \\
\text { son discours. }\end{array}$ & $\begin{array}{l}\text { L: euh les, les chanels } \\
\text { c'est c'est ça? chanel } \\
\text { channel } \\
\text { S: la chaine } \\
\text { L : la chaine }\end{array}$ \\
\hline Production modifiée & $\begin{array}{l}\text { C'est un tour de } \mathrm{R} \text { dans les } \mathrm{NdS} \\
\text { déclenchées par un tour non-conforme de } \\
\text { l'apprenant. L'apprenant modifie sa } \\
\text { production en intégrant la correction de } \\
\text { l'interlocuteur. }\end{array}$ & $\begin{array}{l}\text { L: son compagnie son } \\
\text { propre compagnie } \\
\text { S: sa propre compagnie } \\
\text { L: oui il a sa propre } \\
\text { compagnie }\end{array}$ \\
\hline Rassurement & $\begin{array}{l}\text { C'est un tour de } \mathrm{RR} \text { dans les } \mathrm{NdS} \\
\text { déclenchées par un tour non-conforme de } \\
\text { l'apprenant. Il suit normalement un tour } \\
\text { d'excuse. Le locuteur réconforte } \\
\text { l'interlocuteur. }\end{array}$ & $\begin{array}{l}S \text { : [en riant] non mais } \\
\text { non non non c'est pas } \\
\text { grave c'est pour toi }\end{array}$ \\
\hline Reformulation & $\begin{array}{l}\text { Le locuteur répète l'énoncé, en entier ou } \\
\text { une partie, de l'interlocuteur en corrigeant } \\
\text { une erreur. }\end{array}$ & $\begin{array}{l}\text { L : son compagnie son } \\
\text { propre compagnie } \\
\text { S : sa propre compagnie } \\
\text { L: oui il a sa propre } \\
\text { compagnie }\end{array}$ \\
\hline
\end{tabular}




\begin{tabular}{|c|c|c|}
\hline Réponse minimale & $\begin{array}{l}\text { C'est un tour de R. Le locuteur très } \\
\text { brièvement confirme (ou non) après que } \\
\text { l'interlocuteur a performé une demande de } \\
\text { confirmation. }\end{array}$ & $\begin{array}{l}\mathrm{L}: \text { 没有的意思 } \\
L: \text { il n'y a pas de sens } \\
\mathrm{S}: \text { 没有? } \\
S: \text { il n'y a pas? } \\
\mathrm{L}: \text { 对 } \\
L: \text { oui } \\
\mathrm{S}: \text { ah 好 } \\
S: \text { ah d'accord }\end{array}$ \\
\hline $\begin{array}{l}\text { Utilisation } \\
\text { indépendante } \quad \mathrm{du} \\
\text { clavardage }\end{array}$ & $\begin{array}{l}\text { Le locuteur utilise le clavardage sans rien } \\
\text { dire. }\end{array}$ & \\
\hline $\begin{array}{l}\text { Vérification de } \\
\text { compréhension }\end{array}$ & $\begin{array}{l}\text { Le locuteur demande à son interlocuteur } \\
\text { s'il comprend. }\end{array}$ & $\begin{array}{l}\text { L : les autres c'est l- l'essai } \\
\text { d'accord? }\end{array}$ \\
\hline
\end{tabular}

\section{Tableaux avec les analyses complètes}

$\mathrm{NdS}$ déclenchées par un tour de l'usagère experte

\begin{tabular}{|l|l|l|l|l|}
\hline & \multicolumn{2}{|l|}{ français } & \multicolumn{2}{l|}{ chinois } \\
\hline Déclencheurs & $\mathbf{1 4}$ & $\mathbf{1 0 0} \%$ & $\mathbf{5 1}$ & $\mathbf{1 0 0 \%}$ \\
\hline Lexique & 10 & $71,4 \%$ & 41 & $80,4 \%$ \\
\hline Prononciation et ton & 3 & $21,4 \%$ & 10 & $19,6 \%$ \\
\hline Autre & 1 & $7,2 \%$ & & \\
\hline Signaux & $\mathbf{1 6}$ & $\mathbf{1 0 0} \%$ & $\mathbf{8 3}$ & $\mathbf{1 0 0 \%}$ \\
\hline Alternance codique & & & 5 & $6 \%$ \\
\hline Assertion d'incompréhension & 2 & $12,5 \%$ & 10 & $12 \%$ \\
\hline Demande de clarification & 9 & $56,2 \%$ & 33 & $39,8 \%$ \\
\hline Demande de confirmation & 2 & $12,5 \%$ & 4 & $4,8 \%$ \\
\hline Hétéro-répétition & 3 & $18,8 \%$ & 26 & $31,4 \%$ \\
\hline Non-verbal & 3 & $10,4 \%$ & 34 & $23,3 \%$ \\
\hline Réponses & 4 & $13,8 \%$ & 19 & $13 \%$ \\
\hline Alternance codique & $\mathbf{2 9}$ & $\mathbf{1 0 0} \%$ & $\mathbf{1 4 6}$ & $\mathbf{1 0 0 \%}$ \\
\hline Auto-répétition & & 5 & $6 \%$ \\
\hline
\end{tabular}




\begin{tabular}{|l|l|l|l|l|}
\hline Clavardage & & & 4 & $2,7 \%$ \\
\hline Excuse & 1 & $3,4 \%$ & & \\
\hline Non-verbal & & & 1 & $0,7 \%$ \\
\hline Paraphrase & 13 & $44,8 \%$ & 62 & $42,5 \%$ \\
\hline Réponse minimale & 4 & $13,8 \%$ & 18 & $12,3 \%$ \\
\hline Vérification de compréhension & 4 & $13,8 \%$ & 8 & $5,5 \%$ \\
\hline Réactions aux Réponses & $\mathbf{2 0}$ & $\mathbf{1 0 0} \%$ & $\mathbf{6 7}$ & $\mathbf{1 0 0 \%}$ \\
\hline Accusé de réception & 10 & $50 \%$ & 40 & $59,7 \%$ \\
\hline Alternance codique & 4 & $20 \%$ & & \\
\hline Non-verbal & 1 & $5 \%$ & & \\
\hline Paraphrase & 1 & $5 \%$ & 5 & $7,5 \%$ \\
\hline Prise & 4 & $20 \%$ & 20 & $29,8 \%$ \\
\hline Autre & & & 2 & $3 \%$ \\
\hline
\end{tabular}

NdS déclenchées par un manque dans le tour de l'apprenante

\begin{tabular}{|l|l|l|l|l|}
\hline & \multicolumn{2}{|l}{ français } & \multicolumn{2}{l|}{ chinois } \\
\hline Déclencheurs & 32 & $\mathbf{1 0 0} \%$ & 27 & $\mathbf{1 0 0} \%$ \\
\hline Lexique & 31 & $96,7 \%$ & 27 & $100 \%$ \\
\hline Prononciation & 1 & $3,3 \%$ & & \\
\hline Signaux & 53 & $\mathbf{1 0 0} \%$ & $\mathbf{5 0}$ & $\mathbf{1 0 0} \%$ \\
\hline Alternance codique & 6 & $11,3 \%$ & 22 & $44 \%$ \\
\hline Demande d'aide langagière & 17 & $32,1 \%$ & 14 & $28 \%$ \\
\hline Demande de clarification & 5 & $9,4 \%$ & 8 & $16 \%$ \\
\hline Demande de confirmation & 21 & $39,6 \%$ & 3 & $6 \%$ \\
\hline Non-verbal & 2 & $3,8 \%$ & 3 & $6 \%$ \\
\hline Autre & 2 & $3,8 \%$ & & \\
\hline Réponses & $\mathbf{1 2 2}$ & $\mathbf{1 0 0} \%$ & $\mathbf{1 3 4}$ & $\mathbf{1 0 0 \%}$ \\
\hline
\end{tabular}




\begin{tabular}{|l|l|l|l|l|}
\hline Accomplissement interactif & & & 1 & $0,7 \%$ \\
\hline Alternance codique & 12 & $9,8 \%$ & 36 & $26,9 \%$ \\
\hline Clavardage & 1 & $0,8 \%$ & 3 & $2,2 \%$ \\
\hline Demande de clarification & 15 & $12,3 \%$ & 11 & $8,2 \%$ \\
\hline Hétéro-répétition & 14 & $11,5 \%$ & 17 & $12,7 \%$ \\
\hline Paraphrase & 39 & $32 \%$ & 52 & $38,9 \%$ \\
\hline Reformulation & 9 & $7,4 \%$ & 2 & $1,5 \%$ \\
\hline Réponse minimale & 22 & $18 \%$ & 11 & $8,2 \%$ \\
\hline Vérification de compréhension & 3 & $2,5 \%$ & 1 & $0,7 \%$ \\
\hline Autre & 7 & $5,7 \%$ & & \\
\hline Réactions aux Réponses & $\mathbf{3 3}$ & $\mathbf{1 0 0} \%$ & $\mathbf{3 9}$ & $\mathbf{1 0 0} \%$ \\
\hline Accusé de réception & 18 & $54,5 \%$ & 15 & $38,5 \%$ \\
\hline Prise & 15 & $45,5 \%$ & 21 & $53,8 \%$ \\
\hline Autre & & & 3 & $7,7 \%$ \\
\hline
\end{tabular}

NdS déclenchées par un tour de l'apprenante non-conforme

\begin{tabular}{|l|l|l|l|l|}
\hline & \multicolumn{2}{l|}{ français } & \multicolumn{2}{l|}{ lhinois } \\
\hline Déclencheurs & 36 & $100 \%$ & 30 & $100 \%$ \\
\hline Lexique & 17 & $47,2 \%$ & 14 & $46,7 \%$ \\
\hline Morphosyntaxe & 15 & $41,7 \%$ & 4 & $13,3 \%$ \\
\hline Prononciation & 4 & $11,1 \%$ & 1 & $3,3 \%$ \\
\hline Ton & & & 11 & $36,7 \%$ \\
\hline Signaux & 61 & $100 \%$ & 94 & $100 \%$ \\
\hline Alternance codique & & & 2 & $2,1 \%$ \\
\hline Assertion d'incompréhension & & & 5 & $5,3 \%$ \\
\hline Demande de clarification & 10 & $16,4 \%$ & 15 & $16 \%$ \\
\hline Demande de confirmation & 8 & $13,1 \%$ & 13 & $13,8 \%$ \\
\hline
\end{tabular}




\begin{tabular}{|l|l|l|l|l|}
\hline Explication & 4 & $6,6 \%$ & 30 & $32 \%$ \\
\hline Hétéro-répétition & 10 & $16,4 \%$ & 7 & $7,4 \%$ \\
\hline Non-verbal & & & 2 & $2,1 \%$ \\
\hline Reformulation & 29 & $47,5 \%$ & 20 & $21,3 \%$ \\
\hline Réponses & 61 & $100 \%$ & 85 & $100 \%$ \\
\hline Alternance codique & & & 6 & $7,1 \%$ \\
\hline Auto-répétition & 9 & $14,8 \%$ & 15 & $17,6 \%$ \\
\hline Clavardage & 3 & $11,5 \%$ & 1 & $3,4 \%$ \\
\hline Demande de confirmation & & & 1 & $1,2 \%$ \\
\hline Excuse & 21 & $80,8 \%$ & 26 & $89,7 \%$ \\
\hline Non-verbal & 2 & $3,3 \%$ & 1 & $1,2 \%$ \\
\hline Paraphrase & 1 & $1,6 \%$ & 3 & $3,5 \%$ \\
\hline Production modifiée & $21,9 \%$ & $13,1 \%$ & 9 & $10,6 \%$ \\
\hline Réponse minimale & 3 & $4,9 \%$ & 32 & $37,6 \%$ \\
\hline Vérification de compréhension & $100 \%$ & 29 & $100 \%$ \\
\hline Réactions aux Réponses & & & $14,1 \%$ \\
\hline Accusé de réception & & & $6,9 \%$ \\
\hline Non-verbal & & & \\
\hline Rassurement & & & & \\
\hline
\end{tabular}

\section{NOTES}

1. Nous reprenons, dans notre terminologie, la distinction opérée par Mangenot (2006) entre vidéoconférence et visioconférence, cette dernière indiquant des échanges poste à poste, éventuellement en binôme.

2. La dénomination « cadre input-interaction » vient de Véronique (2011), qui se réfère par là aux travaux de Long sur l'Interaction Hypothesis (Long, 1996) et de Gass (1997). Nous préférons éviter la dénomination "approche(s) interactionniste(s)», car il nous semble qu'elle peut générer une confusion avec ce que Pekarek-Doehler appelle "approches interactionnistes (fortes)", approches s'opposant justement au cadre input-interaction (Pekarek-Doehler, 2000 : par. 10).

3. Notons en passant que la différence entre télétandem et Télé-Tandem (Macaire, 2004) reflète celle entre visioconférence et vidéoconférence (Mangenot, 2006). Autrement dit, si le TéléTandem se déroule entre des groupes d'étudiants, le télétandem implique des binômes. 
4. Nous adoptons la terminologie de ces études, tout en soulignant que nous ne prenons pas à notre compte l' "idéologie du locuteur natif" (Firth \& Wagner, 1997).

5. Les différents types de tour de parole sont définis et illustrés dans l'annexe 2.

6. Pour une analyse approfondie techno-centrée et en termes de potentialités, voir Wang 2006. Pour une analyse dans une perspective de sémiotique sociale, voir Lamy et Flewitt (in Develotte et al., 2011).

7. www.teletandembrasil.org

8. Les cursus de licence en langues en Chine sont de quatre ans.

9. http://www.skype.com

10. Pendant les interactions, environ trente minutes ont été perdues à cause de problèmes techniques.

11. La convention de transcription est présentée dans l'annexe 1.

12. Faute d'espace, les tableaux avec les analyses complètes des NdS sont présentés dans l'annexe 3.

13. Cette notion est définie dans l'annexe 2 .

14. Le chinois mandarin est une langue tonale, où un changement de ton comporte un changement de référent. Par exemple le mot « ma » au premier ton peut signifier « maman » (妈) et au troisième ton « cheval» (马).

15. Voir les annexes pour les normes de transcriptions.

16. Cette notion est définie dans l'annexe 2.

17. Nous ne prenons pas en considération l'analyse que ces auteurs font de la CMO écrite asynchrone, trop différente de la visioconférence.

18. En chinois il y a plus de productions modifiées que de déclencheurs car, après une première production modifiée par l'apprenante, l'usagère experte interrompt parfois l'apprenante avec de nouvelles explications, ce qui amène cette dernière à répéter la production modifiée une deuxième fois quand elle reprend la conversation.

19. En chinois le mot 卡 peut signifier "carte"” ou, en langage familier, "coincé" ou "lent" pour les ordinateurs.

\section{RÉSUMÉS}

Cet article analyse les interactions par visioconférence d'apprenants en tandem étudiant le français et le chinois langues étrangères. Notre but est de comprendre les dynamiques d'acquisition par l'observation des routines de négociations du sens. Le tandem par visioconférence est un type de télécollaboration (Belz \& Thorne, 2006) où deux apprenants de langue maternelle différente interagissent pour s'entraider réciproquement dans leurs apprentissages (Helmling, 2002). Nous adoptons les perspectives du cadre input-interaction (Gass, 1997) et de l'analyse des interactions multimodales par vidéoconférence (Develotte et al., 2011) afin d'étudier trois types de négociations du sens dans les deux langues.

This paper focuses on interactions through desktop videoconference between learners in a tandem studying French and Chinese as a foreign languages. The aim of this study is to characterize language acquisition as it is visible through negotiation of meaning routines. Tandem through desktop videoconference is a type of telecollaboration (Belz \& Thorne, 2006) where two learners of different mother tongues cooperatively learn each other's language 
(Helmling, 2002). We adopt an approach combining tools from the input-interaction framework (Gass, 1997) and the study of multimodal interactions through desktop videoconference (Develotte et al., 2011) in order to study three different types of negotiations of meaning in both languages.

\section{INDEX}

Mots-clés : tandem, visioconférence, négociation du sens, affordances communicatives Keywords : tandem, desktop videoconference, negotiation of meaning, communicative affordances

\section{AUTEURS}

\section{MARCO CAPPELLINI}

Marco Cappellini est doctorant au laboratoire Savoirs Textes Langage (UMR 8163 CNRS) et Attaché Temporaire d'Enseignement et de Recherche à l'Université Lille 3 SHS, UFR Humanités, Département de Sciences du Langage. Ses recherches portent sur l'apprentissage des langues en tandem et sur l'autonomie de l'apprenant.

marco.cappellini@univ-lille3.fr

http://perso.univ-lille3.fr/ mcappellini/

Université Lille 3 - Bât. B, Bureau B4.132

Rue du Barreau - BP 60149

59653 Villeneuve d'Ascq Cedez

\section{MENG ZHANG}

Meng Zhang est chargée de cours à l'UFR de Langues Étrangères Appliquées de l'Université Lille 3 SHS. Après un parcours universitaire dans le Français Langue Étrangère, elle s'intéresse à la didactique du Chinois Langue Étrangère.

meng.zhang2@univ-lille3.fr

UFR des Langues Étrangères Appliquées

14 Place Bodart Timal - BP 447

59058 Rubaix cedex 1 